\title{
THE BERGMAN KERNEL: EXPLICIT FORMULAS, DEFLATION, LU QI-KENG PROBLEM AND JACOBI POLYNOMIALS
}

\author{
Tomasz Beberok
}

In this paper we investigate the Bergman kernel function for intersection of two complex ellipsoids

$$
\left\{\left(z, w_{1}, w_{2}\right) \in \mathbb{C}^{n+2}:\left|z_{1}\right|^{2}+\cdots+\left|z_{n}\right|^{2}+\left|w_{1}\right|^{q}<1, \quad\left|z_{1}\right|^{2}+\cdots+\left|z_{n}\right|^{2}+\left|w_{2}\right|^{r}<1\right\} .
$$

Keyword: Lu Qi-Keng problem, Bergman kernel, Routh-Hurwitz theorem, Jacobi polynomials

AMS Subject Classifications: 32A25; 33D70

\section{Introduction}

Let $D$ be a bounded domain in $\mathbb{C}^{n}$. The Bergman space $L_{a}^{2}(D)$ is the space of all square integrable holomorphic functions on $D$. Then the Bergman kernel $K_{D}(z, w)$ is defined [2] by

$$
K_{D}(z, w)=\sum_{j=0}^{\infty} \Phi_{j}(z) \overline{\Phi_{j}(w)}, \quad(z, w) \in D \times D
$$

where $\left\{\Phi_{j}(\cdot): j=0,1,2, \ldots\right\}$ is a complete orthonormal basis for $L_{a}^{2}(D)$. It is defined for arbitrary domains, but it is hard to obtain concrete representations for the Bergman kernel except for special cases like a Hermitian ball or polydisk. Refer to [6] for more on this topic.

In 2015 [1 the author of this paper computed the Bergman kernel for $D_{1}^{q, r}=$ $\left\{z=\left(z_{1}, w_{1}, w_{2}\right) \in \mathbb{C}^{3}:\left|z_{1}\right|^{2}+\left|w_{1}\right|^{q}<1, \quad\left|z_{1}\right|^{2}+\left|w_{2}\right|^{r}<1\right\}$ explicitly. The goal of this paper is to extend the result in [1] to higher dimensional case, namely to $D_{n}^{q, r}=$ $\left\{\left(z, w_{1}, w_{2}\right) \in \mathbb{C}^{n+2}:\left|z_{1}\right|^{2}+\cdots+\left|z_{n}\right|^{2}+\left|w_{1}\right|^{q}<1, \quad\left|z_{1}\right|^{2}+\cdots+\left|z_{n}\right|^{2}+\left|w_{2}\right|^{r}<1\right\}$.

This paper will be organized as follows. In Section 2, we will compute explicit formula of the Bergman kernel for $D_{n}^{q, r}$ and we show deflation identity between domains $D_{n}^{q, r}$ and $D_{1 / n}^{q, r}=\left\{z=\left(z_{1}, w_{1}, w_{2}\right) \in \mathbb{C}^{3}:\left|z_{1}\right|^{2 / n}+\left|w_{1}\right|^{q}<\right.$ 1, $\left.\left|z_{1}\right|^{2 / n}+\left|w_{2}\right|^{r}<1\right\}$. In Section 3, we show some relation between Bergman kernel for $D_{n}^{2,2}$ and Jacobi polynomials. In Section 4, we investigate the Lu QiKeng problem for $D_{n}^{2,2}$. In the final section, we consider the Bergman kernel for $\Omega_{n}^{r}:=\left\{(z, w) \in \mathbb{C} \times \mathbb{C}^{n}:|z|^{2}+\left|w_{1}\right|^{r}<1, \ldots,|z|^{2}+\left|w_{n}\right|^{r}<1\right\}$. 


\section{Bergman Kernel}

Let $\zeta=\left(z, w_{1}, w_{2}\right) \in \mathbb{C}^{n+2}$. Put $\Phi_{\kappa}(\zeta)=\zeta^{\kappa}=z^{\alpha} w_{1}^{\gamma_{1}} w_{2}^{\gamma_{2}}$ for each multi-index $\kappa=\left(\alpha, \gamma_{1}, \gamma_{2}\right)$. Since $D_{n}^{q, r}$ is complete Reinhardt domain, the collection of $\left\{\Phi_{\kappa}\right\}$ such that each $\alpha_{i} \geq 0$ and $\gamma_{j} \geq 0$ is a complete orthogonal set for $L^{2}\left(D_{n}^{q, r}\right)$.

Proposition 2.1 Let $\alpha_{i} \in \mathbb{Z}_{+}$for $i=1, \ldots, n$ and $\gamma_{1} \geq 0, \gamma_{2} \geq 0$. Then, we have

$$
\left\|z^{\alpha} w_{1}^{\gamma_{1}} w_{2}^{\gamma_{2}}\right\|_{L^{2}\left(D_{n}^{q, r}\right)}^{2}=\frac{\pi^{n+2} \Gamma\left(\frac{2 \gamma_{1}+2}{q}+\frac{2 \gamma_{2}+2}{r}+1\right) \prod_{i=1}^{n} \Gamma\left(\alpha_{i}+1\right)}{\left(\gamma_{2}+1\right)\left(\gamma_{2}+1\right) \Gamma\left(\frac{2 \gamma_{1}+2}{q}+\frac{2 \gamma_{2}+2}{r}+|\alpha|+n+1\right)},
$$

where $|\alpha|=\alpha_{1}+\cdots+\alpha_{n}$.

Proof.

$$
\left\|z^{\alpha} w_{1}^{\gamma_{2}} w_{2}^{\gamma_{3}}\right\|_{L^{2}\left(D_{n}^{q, r}\right)}^{2}=\int_{D_{n}^{q, r}}|z|^{2 \alpha}\left|w_{1}\right|^{2 \gamma_{2}}\left|w_{2}\right|^{2 \gamma_{3}} d V(z) d V(w)
$$

We introduce polar coordinate in each variable by putting $z=r e^{i \theta}, w_{1}=s_{1} e^{i \lambda_{1}}$, $w_{2}=s_{2} e^{i \lambda_{2}}$. After doing so, and integrating out the angular variables we have

$$
(2 \pi)^{n+2} \int_{\operatorname{Re}\left(D_{n}^{q, r}\right)} r^{2 \alpha+1} s_{1}^{2 \gamma_{1}+1} s_{2}^{2 \gamma_{2}+1} d V(r) d V(s),
$$

where $\operatorname{Re}\left(D_{n}^{q, r}\right)=\left\{(r, s) \in \mathbb{R}_{+}^{n} \times \mathbb{R}_{+}^{2}:\|r\|^{2}+s_{1}^{q}<1, \quad\|r\|^{2}+s_{2}^{r}<1\right\}$. Next we use spherical coordinates in the $r$ variable to obtain

$$
(2 \pi)^{n+2} \int_{S * \operatorname{Re}\left(D_{n}^{q, r}\right)} \int_{\mathbf{S}_{+}^{n_{1}-1}} \rho^{2|\alpha|+2 n-1} \omega^{2 \alpha+1} s_{1}^{2 \gamma_{1}+1} s_{2}^{2 \gamma_{2}+1} d \sigma(\omega) d V(s) d \rho,
$$

where $S * \operatorname{Re}\left(D_{n}^{q, r}\right)=\left\{(\rho, s) \in \mathbb{R}_{+} \times \mathbb{R}_{+}^{2}: \rho^{2}+s_{1}^{q}<1, \quad \rho^{2}+s_{2}^{r}<1\right\}$. Using (6) form Lemma 1 in [4] we obtain

$$
(2 \pi)^{n+2} \frac{\beta(\alpha+1)}{2^{n-1}} \int_{0}^{1} \int_{0}^{\left(1-\rho^{2}\right)^{1 / q}} \int_{0}^{\left(1-\rho^{2}\right)^{1 / r}} \rho^{2|\alpha|+2 n-1} s_{1}^{2 \gamma_{1}+1} s_{2}^{2 \gamma_{2}+1} d s_{1} d s_{2} d \rho,
$$

where $\beta(\alpha)=\frac{\prod_{i=1}^{n} \Gamma\left(\alpha_{i}\right)}{\Gamma\left(\alpha_{1}+\cdots+\alpha_{n}\right)}$. Integrating out of $s_{1}$ and $s_{2}$ variables, we have

$$
\frac{(2 \pi)^{n+2} \beta(\alpha+1)}{2^{n+1}\left(\gamma_{1}+1\right)\left(\gamma_{2}+1\right)} \int_{0}^{1} \rho^{2|\alpha|+2 n-1}\left(1-\rho^{2}\right)^{\frac{2 \gamma_{1}+2}{q}+\frac{2 \gamma_{2}+2}{r}} d \rho
$$

After a little calculation using well known fact

$$
\int_{0}^{1} x^{a}\left(1-x^{p}\right)^{b} d x=\frac{\Gamma((a+1) / p) \Gamma(b+1)}{p \Gamma((a+1) / p+b+1)},
$$

we obtain desired result.

Now we discuss the Bergman kernel for $D_{n}^{q, r}$. 
Theorem 2.1 The Bergman kernel for $D_{n}^{q, r}$ is given by

$$
K_{D_{n}^{q, r}}\left(\left(z, w_{1}, w_{2}\right),\left(\eta, \xi_{1}, \xi_{2}\right)\right)=\frac{L_{n}^{q, r}(a, b)}{\pi^{n+2}\left(1-z_{1} \bar{\eta}_{1}-\cdots-z_{n} \bar{\eta}_{n}\right)^{\frac{2}{q}+\frac{2}{r}+1}},
$$

where

$$
\begin{array}{r}
a=\frac{w_{1} \bar{\xi}_{1}}{\left(1-z_{1} \bar{\eta}_{1}-\cdots-z_{n} \bar{\eta}_{n}\right)^{\frac{2}{q}}}, \quad b=\frac{w_{2} \bar{\xi}_{2}}{\left(1-z_{1} \bar{\eta}_{1}-\cdots-z_{n} \bar{\eta}_{n}\right)^{\frac{2}{r}}} \\
L_{n+1}^{q, r}(x, y)=n L_{n}^{q, r}(x, y)+\frac{2}{q} \frac{\partial}{\partial x} x L_{n}^{q, r}(x, y)+\frac{2}{r} \frac{\partial}{\partial y} y L_{n}^{q, r}(x, y) \text { and } \\
L_{1}^{q, r}(x, y)=\frac{2(q(1-x)(y+1)+r(x+1)(1-y))}{q r(1-x)^{3}(1-y)^{3}}
\end{array}
$$

Proof. By Proposition 2.1, we have

$$
\begin{aligned}
& K_{D_{n}^{q, r}}\left(\left(z, w_{1}, w_{2}\right),\left(\eta, \xi_{1}, \xi_{2}\right)\right) \\
& =\frac{1}{\pi^{n+2}} \sum_{\alpha=0}^{\infty} \sum_{\gamma_{1}, \gamma_{2}=0}^{\infty} \frac{\left(\gamma_{1}+1\right)\left(\gamma_{2}+1\right) \Gamma\left(\frac{2 \gamma_{1}+2}{q}+\frac{2 \gamma_{2}+2}{r}+|\alpha|+n+1\right)}{\Gamma\left(\frac{2 \gamma_{1}+2}{q}+\frac{2 \gamma_{2}+2}{r}+1\right) \prod_{i=1}^{n} \Gamma\left(\alpha_{i}+1\right)} \mu^{\alpha} \nu_{1}^{\gamma_{1}} \nu_{2}^{\gamma_{2}},
\end{aligned}
$$

where $\mu^{\alpha}=\left(z_{1} \bar{\eta}_{1}\right)^{\alpha_{1}} \ldots .\left(z_{n} \bar{\eta}_{n}\right)^{\alpha_{n}}$ and $\nu_{1}=w_{1} \bar{\xi}_{1}, \nu_{2}=w_{2} \bar{\xi}_{2}$. Sum out of each $\alpha_{i}$, we have

$$
\frac{1}{\pi^{n+2}(1-\tau)^{\frac{2}{q}+\frac{2}{r}+1}} \sum_{\gamma_{1}, \gamma_{2}=0}^{\infty} \frac{\left(\gamma_{1}+1\right)\left(\gamma_{2}+1\right) \Gamma\left(\frac{2 \gamma_{1}+2}{q}+\frac{2 \gamma_{2}+2}{r}+n+1\right)}{\Gamma\left(\frac{2 \gamma_{1}+2}{q}+\frac{2 \gamma_{2}+2}{r}+1\right)(1-\tau)^{\frac{2 \gamma_{1}}{q}+\frac{2 \gamma_{2}}{r}}} \nu_{1}^{\gamma_{1}} \nu_{2}^{\gamma_{2}},
$$

where $\tau=z_{1} \bar{\eta}_{1}+\cdots+z_{n} \bar{\eta}_{n}$. Now we will consider sequence of functions $L_{n}^{q, r}$ defined as follows

$$
L_{n}^{q, r}(x, y)=\sum_{\gamma_{1}, \gamma_{2}=0}^{\infty} \frac{\left(\gamma_{1}+1\right)\left(\gamma_{2}+1\right) \Gamma\left(\frac{2 \gamma_{1}+2}{q}+\frac{2 \gamma_{2}+2}{r}+n+1\right)}{\Gamma\left(\frac{2 \gamma_{1}+2}{q}+\frac{2 \gamma_{2}+2}{r}+1\right)} x^{\gamma_{1}} y^{\gamma_{2}}
$$

Using the identity $\Gamma(t+1)=t \Gamma(t)$ we easily obtain recursion formula

$$
L_{n+1}^{q, r}(x, y)=n L_{n}^{q, r}(x, y)+\frac{2}{q} \frac{\partial}{\partial x} x L_{n}^{q, r}(x, y)+\frac{2}{r} \frac{\partial}{\partial y} y L_{n}^{q, r}(x, y)
$$

Moreover

$$
L_{1}^{q, r}(x, y)=\sum_{\gamma_{1}, \gamma_{2}=0}^{\infty}\left(\gamma_{1}+1\right)\left(\gamma_{2}+1\right)\left(\frac{2 \gamma_{1}+2}{q}+\frac{2 \gamma_{2}+2}{r}+1\right) x^{\gamma_{1}} y^{\gamma_{2}}
$$

Hence

$$
L_{1}^{q, r}(x, y)=\frac{2(q(1-x)(y+1)+r(x+1)(1-y))}{q r(1-x)^{3}(1-y)^{3}}
$$

which completes the proof. 


\subsection{Deflation}

Now we will consider following domains

$$
D_{1 / n}^{q, r}=\left\{\left(z, w_{1}, w_{2}\right) \in \mathbb{C}^{3}:|z|^{2 / n}+\left|w_{1}\right|^{q}<1, \quad|z|^{2 / n}+\left|w_{2}\right|^{r}<1\right\}
$$

Similarly as in Sect. 2, we have

Proposition 2.2 Let $\alpha \geq 0, \gamma_{1} \geq 0$ and $\gamma_{2} \geq 0$. Then, we have

$$
\left\|z^{\alpha} w_{1}^{\gamma_{1}} w_{2}^{\gamma_{2}}\right\|_{L^{2}\left(D_{1 / n}^{q, r}\right)}^{2}=\frac{n \pi^{3} \Gamma\left(\frac{2 \gamma_{1}+2}{q}+\frac{2 \gamma_{2}+2}{r}+1\right) \Gamma(n \alpha+n)}{\left(\gamma_{2}+1\right)\left(\gamma_{2}+1\right) \Gamma\left(\frac{2 \gamma_{1}+2}{q}+\frac{2 \gamma_{2}+2}{r}+n \alpha+n+1\right)} .
$$

Therefore

$$
\begin{aligned}
& K_{D_{1 / n}^{q, r}}\left(\left(0, w_{1}, w_{2}\right),\left(0, \xi_{1}, \xi_{2}\right)\right) \\
& =\sum_{\gamma_{1}, \gamma_{2}=0}^{\infty} \frac{\left(\gamma_{1}+1\right)\left(\gamma_{2}+1\right) \Gamma\left(\frac{2 \gamma_{1}+2}{q}+\frac{2 \gamma_{2}+2}{r}+n+1\right)}{\pi^{3} n ! \Gamma\left(\frac{2 \gamma_{1}+2}{q}+\frac{2 \gamma_{2}+2}{r}+1\right)}\left(w_{1} \bar{\xi}_{1}\right)^{\gamma_{1}}\left(w_{2} \bar{\xi}_{2}\right)^{\gamma_{2}}
\end{aligned}
$$

Hance

$$
K_{D_{1 / n}^{q, r}}\left(\left(0, w_{1}, w_{2}\right),\left(0, \xi_{1}, \xi_{2}\right)\right)=\frac{1}{\pi^{3} n !} L_{n}^{q, r}\left(w_{1} \bar{\xi}_{1}, w_{2} \bar{\xi}_{2}\right)
$$

Comparing formulas for $K_{D_{1 / n}^{q, r}}$ and $K_{D_{n}^{q, r}}$, we obtain following deflation identity

Proposition 2.3 For every $n \in \mathbb{N}$ and every positive numbers $q$ and $r$, we have

$$
\frac{n !}{\pi^{n-1}} K_{D_{1 / n}^{q, r}}\left(\left(0, w_{1}, w_{2}\right),\left(0, \xi_{1}, \xi_{2}\right)\right)=K_{D_{n}^{q, r}}\left(\left(0, \ldots, 0, w_{1}, w_{2}\right),\left(0, \ldots, 0, \xi_{1}, \xi_{2}\right)\right)
$$

Note that, we have some kind of deflation result similar to that obtained in [3].

\section{Some representations of Bergman kernel for $D_{n}^{2,2}$}

Jacobi polynomials are a class of classical orthogonal polynomials. They are orthogonal with respect to the weight $(1-x)^{k}(1+x)^{l}$ on the interval $[-1,1]$. For $k, l>-1$ the Jacobi polynomials are given by the formula

$$
P_{d}^{(k, l)}(z)=\frac{(-1)^{d}}{2^{d} d !}(1-z)^{-k}(1+z)^{-l} \frac{\partial}{\partial z^{d}}\left\{(1-z)^{k}(1+z)^{l}\left(1-z^{2}\right)^{d}\right\} .
$$

The Jacobi polynomials are defined via the hypergeometric function as follows

$$
P_{d}^{(k, l)}(z)=\frac{(k+1)_{d}}{d !}{ }_{2} F_{1}\left(-d, 1+k+l+d ; k+1, \frac{1-z}{2}\right)
$$

where $(k+1)_{d}$ is Pochhammer's symbol and ${ }_{2} F_{1}$ is Gaussian or ordinary hypergeometric function defined for $|z|<1$ by the power series

$$
{ }_{2} F_{1}(a, b ; c ; z)=\sum_{n=0}^{\infty} \frac{(a)_{n}(b)_{n}}{n !(c)_{n}} z^{n}
$$


Appell series $F_{1}$ defined by

$$
F_{1}\left(a ; b, b^{\prime} ; c, x, y\right)=\sum_{n=0}^{\infty} \sum_{m=0}^{\infty} \frac{(a)_{m+n}(b)_{n}\left(b^{\prime}\right)_{m}}{n ! m !(c)_{n+m}} x^{n} y^{m}, \quad(|x|<1,|y|<1)
$$

is one of a natural two-variable extension of hypergeometric series ${ }_{2} F_{1}$.

The following reduction formulas can be proved (see, for details [5], p. 238-239)

$$
\begin{aligned}
F_{1}\left(a ; b, b^{\prime} ; b+b^{\prime} ; x, y\right) & =(1-y)^{-a}{ }_{2} F_{1}\left(a, b ; b+b^{\prime} ;(x-y) /(1-y)\right) \\
F_{1}\left(a ; b, b^{\prime} ; c ; x, x\right) & ={ }_{2} F_{1}\left(a, b+b^{\prime} ; c ; x\right)
\end{aligned}
$$

Comparing functions $F_{1}$ and $L_{n}^{2,2}$ it is easy to see

$$
\frac{\Gamma(3+n)}{2} F_{1}(3+n ; 2,2 ; 3 ; x, y)=L_{n}^{2,2}(x, y)
$$

The following is the main theorem of this section.

Theorem 3.1 The Bergman kernel for $D_{n}^{2,2}$ can be expressed in the following ways

(i) $K_{D_{n}^{2,2}}\left(\left(0, w_{1}, w_{2}\right),\left(0, \xi_{1}, \xi_{2}\right)\right)=$

$$
\frac{\Gamma(3+n)}{2 \pi^{n+2}} \sum_{i=0}^{n} \sum_{k=0}^{n-i}\left(\begin{array}{c}
n \\
i
\end{array}\right)\left(\begin{array}{c}
n-1 \\
k
\end{array}\right) \frac{(2)_{i}(2)_{k}\left(w_{1} \bar{\xi}_{1}\right)^{i}\left(w_{2} \bar{\xi}_{2}\right)^{k}}{(3)_{i+k}\left(1-w_{1} \bar{\xi}_{1}\right)^{2+i}\left(1-w_{2} \bar{\xi}_{2}\right)^{2+k}}
$$

(ii) For every $m \in \mathbb{N} \cup\{0\}$, we have

$$
\begin{aligned}
& K_{D_{2 m+1}^{2,2}}\left(\left(0, w_{1}, w_{2}\right),\left(0, \xi_{1}, \xi_{2}\right)\right)=\frac{C_{m}(2+m)\left(2-\nu_{1}-\nu_{2}\right) P_{m}^{\left(\frac{3}{2}, \frac{1}{2}\right)}\left(\frac{x^{\prime}+1}{1-x^{\prime}}\right)}{\pi^{2 m+3}\left(1-\nu_{1}-\nu_{2}-\nu_{1} \nu_{2}\right)^{m+3}} \\
& -\frac{C_{m}(2 m+1) P_{m}^{\left(\frac{3}{2},-\frac{1}{2}\right)}\left(\frac{x^{\prime}+1}{1-x^{\prime}}\right)}{\pi^{2 m+3}\left(1-\nu_{1}-\nu_{2}-\nu_{1} \nu_{2}\right)^{m+2}}
\end{aligned}
$$

(iii) For every $m \in \mathbb{N}$, we have $K_{D_{2 m}^{2,2}}\left(\left(0, w_{1}, w_{2}\right),\left(0, \xi_{1}, \xi_{2}\right)\right)=$

$$
\Gamma(3+2 m) m ! \frac{2 P_{m}^{\left(\frac{3}{2},-\frac{1}{2}\right)}\left(\frac{x^{\prime}+1}{1-x^{\prime}}\right)-\left(2-\nu_{1}-\nu_{2}\right) P_{m-1}^{\left(\frac{3}{2}, \frac{1}{2}\right)}\left(\frac{x^{\prime}+1}{1-x^{\prime}}\right)}{\pi^{2 m+2} 6\left(\frac{5}{2}\right)_{m-1}\left(1-\nu_{1}-\nu_{2}-\nu_{1} \nu_{2}\right)^{m+2}},
$$

(iv) If $w_{1} \bar{\xi}_{1}=w_{2} \bar{\xi}_{2}$ then

$$
K_{D_{n}^{2,2}}\left(\left(0, w_{1}, w_{2}\right),\left(0, \xi_{1}, \xi_{2}\right)\right)=\frac{\Gamma(3+n)\left(3+n w_{1} \bar{\xi}_{1}\right)}{6 \pi^{n+2}\left(1-w_{1} \bar{\xi}_{1}\right)^{4+n}}
$$


Proof. For the proof of (i), if we apply the recursion formula (see [8])

$$
\begin{aligned}
F_{1}\left(a+n ; b, b^{\prime} ; c ; x, y\right)= & \sum_{i=0}^{n} \sum_{k=0}^{n-i}\left(\begin{array}{c}
n \\
i
\end{array}\right)\left(\begin{array}{c}
n-1 \\
k
\end{array}\right) \frac{(b)_{i}\left(b^{\prime}\right)_{k}}{(c)_{i+k}} \\
& \times x^{i} y^{k} F_{1}\left(a+i+k ; b+i, b^{\prime}+k ; c+i+k ; x, y\right),
\end{aligned}
$$

then using formula

$$
F_{1}(3+i+k ; 2+i, 2+k ; 3+i+k ; x, y)=\frac{1}{(1-x)^{2+i}(1-y)^{2+k}}
$$

we obtain (i).

In order to prove the second statements we need the following well-known contiguous relation

$$
\begin{aligned}
c F_{1}\left(a ; b, b^{\prime} ; c ; x, y\right)- & (c-a) F_{1}\left(a ; b, b^{\prime} ; c+1 ; x, y\right) \\
& -a F_{1}\left(a+1 ; b, b^{\prime} ; c+1 ; x, y\right)=0
\end{aligned}
$$

It follows that

$$
\begin{aligned}
F_{1}(4+2 m ; 2,2 ; 3 ; x, y)= & -\frac{2 m+1}{3} F_{1}(2 m+4 ; 2,2 ; 4 ; x, y) \\
& +\frac{4+2 m}{3} F_{1}(2 m+5 ; 2,2 ; 4 ; x, y)
\end{aligned}
$$

By (2), we have

$$
\begin{aligned}
F_{1}(4+2 m ; 2,2 ; 3 ; x, y)= & -\frac{2 m+1}{3(1-y)^{4+2 m}}{ }_{2} F_{1}\left(2 m+4,2 ; 4 ; \frac{x-y}{1-y}\right) \\
& +\frac{4+2 m}{3(1-y)^{5+2 m}}{ }_{2} F_{1}\left(2 m+5,2 ; 4 ; \frac{x-y}{1-y}\right)
\end{aligned}
$$

Then by (see, for details [5], p. 66)

$$
{ }_{2} F_{1}(a, b ; 2 b ; z)=\left(1-\frac{z}{2}\right)^{-a}{ }_{2} F_{1}\left(\frac{a}{2}, \frac{a+1}{2} ; b+\frac{1}{2} ;[z /(2-z)]^{2}\right),
$$

we have

$$
\begin{aligned}
F_{1}(4+2 m ; 2,2 ; 3 ; x, y)= & -\frac{(2 m+1) 2^{4+2 m}}{3(2-x-y)^{4+2 m}}{ }_{2} F_{1}\left(m+2, m+\frac{5}{2} ; \frac{5}{2} ;\left(x^{\prime}\right)^{2}\right) \\
& +\frac{(4+2 m) 2^{5+2 m}}{3(2-x-y)^{5+2 m}}{ }_{2} F_{1}\left(m+\frac{5}{2}, m+3 ; \frac{5}{2} ;\left(x^{\prime}\right)^{2}\right)
\end{aligned}
$$

where $x^{\prime}=\left(\frac{x-y}{2-x-y}\right)^{2}$. Next by (see, for details [5], p. 64)

$$
\begin{aligned}
{ }_{2} F_{1}(a, b ; c ; z) & =(1-z)_{2}^{-a} F_{1}(a, c-b ; c ; z /(z-1)) \\
& =(1-z)^{-b}{ }_{2} F_{1}(c-a, b ; c ; z /(z-1)),
\end{aligned}
$$


we can easily get $F_{1}(4+2 m ; 2,2 ; 3 ; x, y)=$

$$
\begin{aligned}
& -\frac{2 m+1}{3(1-x-y-x y)^{m+2}}{ }_{2} F_{1}\left(m+2,-m ; \frac{5}{2} ;\left(\frac{x^{\prime}}{x^{\prime}-1}\right)^{2}\right) \\
& +\frac{(2+m)(2-x-y)}{3(1-x-y-x y)^{m+3}}{ }_{2} F_{1}\left(m+\frac{5}{2}, m+3 ; \frac{5}{2} ;\left(\frac{x^{\prime}}{x^{\prime}-1}\right)^{2}\right),
\end{aligned}
$$

Finally, by (11) we obtain (ii). The formula (iii) can be obtained by the same method and we omit the details.

Now we will prove (iv). This is a formal exercise but we include it for completeness: From (3)

$$
F_{1}(3+n ; 2,2 ; 3 ; x, y)={ }_{2} F_{1}(3+n, 4 ; 3 ; x)
$$

Now it is relatively easy to compute the following result

$$
{ }_{2} F_{1}(3+n, 4 ; 3 ; x)=\frac{3+n x}{3(1-x)^{4+n}}
$$

Thus we prove (iv).

\section{Lu Qi-Keng problem}

The explicit formula of the Bergman kernel function for the domains $D_{n}^{q, r}$ enables us to investigate whether the Bergman kernel has zeros in $D_{n}^{q, r} \times D_{n}^{q, r}$ or not. We will call this kind of problem a Lu Qi-Keng problem. If the Bergman kernel for a bounded domain does not have zeros, then the domain will be called a Lu Qi-Keng domain.

By Theorem 1.2 form [1] if $n=1$, then $D_{n}^{q, r}$ is a Lu Qi-Keng domain for all positive real numbers $q$ and $r$. Combining deflation identity (Proposition 2.3) and Proposition 4.4 from [1], if $n=2$ and $q=r$, then $D_{2}^{r, r}$ is not a Lu Qi-Keng domain. Using the same method as in [1] we will prove that it is also true for $n=3$.

Denote by

$$
\begin{aligned}
G(x, y)= & 3 r^{3}(1-x)^{3}(1-y)^{3}+22 r^{2}(1-x)^{2}(1-y)^{2}(1-x y) \\
& +24 r(1-x)(1-y)\left(x(2 x+1) y^{2}+(x-8) x y+x+y+2\right) \\
& +8(1-x y)\left(x^{2} y(4 y+7)+x^{2}+y^{2}+x y(7 y-38)+7 x+7 y+4\right) .
\end{aligned}
$$

Since

$$
G(x, y)=\frac{r^{3}(1-x)^{5}(1-y)^{5}}{2} L_{3}^{r, r}(x, y)
$$

then the Bergman kernel $K_{D_{3}^{r, r}}$ has zero inside $D_{2}^{r, r} \times D_{2}^{r, r}$ if polynomial $G(\epsilon x, \epsilon y)$ does not satisfy the stability property (see [1], for details) for some $0<\epsilon<1$. By following Šiljak and Stipanović [7] we consider polynomial

$$
z^{3} G\left(e^{i \eta}, 1 / z\right)=d(z)=d_{3} z^{3}+d_{2} z^{2}+d_{1} z+d_{0},
$$


where

$$
\begin{aligned}
& d_{0}= 3 r^{3}(-1+t)^{3}-22 r^{2}(-1+t)^{2} t+24 r t\left(-1-t+2 t^{2}\right)-8 t\left(1+7 t+4 t^{2}\right), \\
& d_{1}= 8-9 r^{3}(-1+t)^{3}+336 t^{2}-56 t^{3}+22 r^{2}(-1+t)^{2}(1+2 t) \\
&-24 r\left(1-10 t+8 t^{2}+t^{3}\right), \\
& d_{2}= 9 r^{3}(-1+t)^{3}-22 r^{2}\left(2-3 t+t^{3}\right)-8\left(-7+42 t+t^{3}\right) \\
&-24 r\left(1+8 t-10 t^{2}+t^{3}\right), \\
& d_{3}= 22 r^{2}(-1+t)^{2}-3 r^{3}(-1+t)^{3}-24 r\left(-2+t+t^{2}\right)+8\left(4+7 t+t^{2}\right) \\
& \text { and } \quad t=e^{i \eta} .
\end{aligned}
$$

With the polynomial $d(z)$ we associate the Schur-Cohn $3 \times 3$ matrix

$$
M\left(e^{i \eta}\right)=\left(\begin{array}{lll}
d_{11} & d_{12} & d_{13} \\
d_{21} & d_{22} & d_{23} \\
d_{31} & d_{32} & d_{33}
\end{array}\right)
$$

$d_{j k}=\sum_{l=1}^{j}\left(d_{m-j+l} \bar{d}_{m-k+l}-\bar{d}_{j-l} d_{k-l}\right)$, where $1 \leq j \leq k$. The matrix $M\left(e^{i \eta}\right)$ is defined when $j>k$ to become Hermitian. After some calculation (with the help of a computer program Maple or Mathematica), we have

$$
\operatorname{det} M\left(e^{i \eta}\right)=-130459631616 r^{3} \sin ^{12}(\eta / 2)\left[\sum_{n=0}^{3} g_{n}(r) \cos (n \eta)\right]
$$

where

$$
\begin{aligned}
& g_{0}(r)=26624-24672 r^{2}+15724 r^{4}-2430 r^{6} \\
& g_{1}(r)=12288+22496 r^{2}-20822 r^{4}+3645 r^{6} \\
& g_{2}(r)=-512+2208 r^{2}+5012 r^{4}-1458 r^{6} \\
& g_{3}(r)=r^{2}\left(-32+86 r^{2}+243 r^{4}\right)
\end{aligned}
$$

Since

$$
\sum_{n=0}^{3} g_{n}(r)=38400
$$

Therefore it is easy to see that for every $r>0$ there exist $\eta$ such that $\operatorname{det} M\left(e^{i \eta}\right)<$ 0 . Hence there exist $1>\epsilon>0$, such that polynomial $G(\epsilon x, \epsilon y)$ does not satisfy the stability property (see [7]). As a consequence of above consideration, we have following corollary

Corollary 4.1 For any $r>0$, domains $D_{3}^{r, r}$ and $D_{1 / 3}^{r, r}$ are not Lu Qi-Keng.

Now we will study Lu Qi-Keng problem for $D_{n}^{2,2}$ in the case when $n>3$. By representation (iv) from Theorem 3.1

$$
K_{D_{n}^{2,2}}\left(\left(0, w_{1}, w_{1}\right),\left(0, \xi_{1}, \xi_{1}\right)\right)=\frac{\Gamma(3+n)\left(3+n w_{1} \bar{\xi}_{1}\right)}{6 \pi^{n+2}\left(1-w_{1} \bar{\xi}_{1}\right)^{4+n}} .
$$


Hence

$$
K_{D_{n}^{2,2}}((0, i \sqrt{3 / n}, i \sqrt{3 / n}),(0,-i \sqrt{3 / n},-i \sqrt{3 / n}))=0
$$

For brevity, we shall summarize these last statements by saying that

Proposition 4.1 Domain $D_{n}^{2,2}$ is Lu Qi-Keng if and only if $n=1$.

At the end of this section we would like to present following relations between zeros of the Bergman kernel for domains $D_{n}^{q, r}$ and $D_{1 / n}^{q, r}$.

Proposition 4.2 For any positive real numbers $q$ and $r$

(i) If $K_{D_{n}^{q, r}}$ has zeros, then $K_{D_{1 / n}^{q, r}}$ also has zeros,

(ii) If $D_{1 / n}^{q, r}$ is Lu Qi-Keng domain, then $D_{n}^{q, r}$ is Lu Qi-Keng domain.

Proof. Note that the zero set is a bi-holomorphic invariant object. Since any point $\left(z, w_{1}, w_{2}\right) \in D_{n}^{q, r}$ can be mapped equivalently onto the form $\left(0, \widetilde{w_{1}}, \widetilde{w_{2}}\right)$ by following automorphism of the $D_{n}^{q, r}$

$$
D_{n}^{q, r} \ni\left(z, w_{1}, w_{2}\right) \mapsto\left(\Psi_{a}(z), \frac{\left(1-\|a\|^{2}\right)^{1 / q}}{(1-\langle z, a\rangle)^{2 / q}} w_{1}, \frac{\left(1-\|a\|^{2}\right)^{1 / r}}{(1-\langle z, a\rangle)^{2 / r}} w_{2}\right) \in \mathbb{C}^{n+2},
$$

where

$$
\Psi_{a}(z)=\frac{\left(\frac{\langle z, a\rangle}{1+\sqrt{1-\|a\|^{2}}}-1\right) a+z \sqrt{1-\|a\|^{2}}}{1-\langle z, a\rangle} .
$$

Therefore, we need only consider the zeroes restricted to $\{0\} \times \mathbb{D} \times \mathbb{D}$, where $\mathbb{D}:=\{z \in \mathbb{C}:|z|<1\}$. The results now follows from Proposition 2.3 ,

\section{$5 \quad$ Additional Results}

Our purpose in this section is to consider domains $\Omega_{n}^{r}$ defined for every positive real number $r$ by

$$
\Omega_{n}^{r}:=\left\{(z, w) \in \mathbb{C} \times \mathbb{C}^{n}:|z|^{2}+\left|w_{1}\right|^{r}<1, \ldots,|z|^{2}+\left|w_{n}\right|^{r}<1\right\}
$$

The reader can see that following proposition is completely analogous to the results presented earlier.

Proposition 5.1 For $j=1,2, \ldots, n$ let $\beta_{j} \geq 0$. Then for $\alpha \geq 0$, we have

$$
\left\|z^{\alpha} w^{\beta}\right\|_{L^{2}\left(\Omega_{n}\right)}^{2}=\frac{\pi^{n+1} \Gamma\left(\frac{2}{r}\left(\sum_{j=1}^{n} \beta_{j}+n\right)+1\right) \Gamma(\alpha+1)}{\Gamma\left(\frac{2}{r}\left(\sum_{j=1}^{n} \beta_{j}+n\right)+\alpha+2\right) \prod_{j=1}^{n}\left(\beta_{j}+1\right)} .
$$

Now we will give an explicit formula for the kernel $K_{\Omega_{n}^{r}}$ of $\Omega_{n}^{r}$. 
Theorem 5.1 If $w, \zeta \in \mathbb{D}^{n}$, then the Bergman kernel for $\Omega_{n}^{r}$ is

$$
\pi^{n+1} K_{\Omega_{n}^{r}}((0, w),(0, \zeta))=\prod_{k=1}^{n}\left(1-\nu_{k}\right)^{-2}+\sum_{k=1}^{n} \frac{2\left(1+\nu_{k}\right)}{r\left(1-\nu_{1}\right)^{2} \cdots\left(1-\nu_{n}\right)^{2}\left(1-\nu_{k}\right)},
$$

where $\nu_{k}=w_{k} \bar{\zeta}_{k}$ for $k=1, \ldots, n$. Moreover if $\nu_{1}=\nu_{2}=\cdots=\nu_{n}$, then

$$
\pi^{n+1} K_{\Omega_{n}^{r}}((0, w),(0, \zeta))=\frac{(2 n-r) \nu_{1}+2 n+r}{r\left(1-\nu_{1}\right)^{2 n+1}} .
$$

Proof. As a consequence of Proposition 5.1, we have

$$
\pi^{n+1} K_{\Omega_{n}^{r}}((0, w),(0, \zeta))=\sum_{\beta_{1}, \ldots, \beta_{n} \geq 0}^{\infty} \frac{\Gamma\left(\frac{2}{r}\left(\sum_{j=1}^{n} \beta_{j}+n\right)+2\right) \prod_{j=1}^{n}\left(\beta_{j}+1\right)}{\Gamma\left(\frac{2}{r}\left(\sum_{j=1}^{n} \beta_{j}+n\right)+1\right)} \nu^{\beta}
$$

where $\nu^{\beta}=\nu_{1}^{\beta_{1}} \cdots \cdots \nu_{n}^{\beta_{n}}$. Then, using the fact that $a \Gamma(a)=\Gamma(a+1)$,

$$
\pi^{n+1} K_{\Omega_{n}^{r}}((0, w),(0, \zeta))=\sum_{\beta_{1}, \ldots, \beta_{n} \geq 0}^{\infty} \frac{2}{r}\left(\beta_{1}+\cdots+\beta_{n}+n+\frac{r}{2}\right) \prod_{j=1}^{n}\left(\beta_{j}+1\right) \nu^{\beta} .
$$

Hence, the kernel is

$$
\frac{2}{r \pi^{n+1}} \sum_{k=0}^{n} \sum_{\beta_{1}, \ldots, \beta_{n} \geq 0}^{\infty}\left(\beta_{k}+1\right) \prod_{j=1}^{n}\left(\beta_{j}+1\right) \nu^{\beta}
$$

where $\beta_{0}=r / 2-1$. After some calculation using formulas

$$
\sum_{m=0}^{\infty}(m+1) x^{m}=(1-x)^{-2} \text { and } \quad \sum_{m=0}^{\infty}(m+1)^{2} x^{m}=\frac{1+x}{(1-x)^{3}},
$$

we obtain desired formula.

\subsection{Zeros of Bergman kernels on $\Omega_{n}^{r}$}

In this section, we will prove that the Bergman kernel function of $\Omega_{n}^{r}$ for any natural number $n$ and positive real number $r$ is zero-free.

Proposition 5.2 For any positive integer $n$, the domain $\Omega_{n}^{r}$ is a Lu Qi-Keng domain.

Proof. It is obvious that the Bergman kernel for $\Omega_{1}^{r}$ has no zeros. The Bergman kernel function of $\Omega_{n}^{r}$ has zeros iff

$$
\frac{r}{2}+\sum_{k=1}^{n} \frac{1+\nu_{k}}{1-\nu_{k}}=n+\frac{r}{2}+\sum_{k=1}^{n} \frac{2 \nu_{k}}{1-\nu_{k}}=0
$$


for some $\nu_{1}, \ldots, \nu_{n}$ such that $\left|\nu_{k}\right|<1$, for each $k=1, \ldots, n$. Since $\left|\nu_{k}\right|<1$, then

$$
\Re\left(\frac{2 \nu_{k}}{1-\nu_{k}}\right)>-1
$$

where $\Re(\xi)$ is the real part of complex number $\xi$. Thus

$$
\Re\left(n+\frac{r}{2}+\sum_{k=1}^{n} \frac{2 \nu_{k}}{1-\nu_{k}}\right)>0 .
$$

Hence, we see that

$$
\frac{r}{2}+\sum_{k=1}^{n} \frac{1+\nu_{k}}{1-\nu_{k}} \neq 0,
$$

if $\left(\nu_{1}, \ldots, \nu_{n}\right) \in \mathbb{D}^{n}$. The proof is therefore complete.

\section{References}

[1] T. Beberok, Lu Qi-Keng's Problem for Intersection of Two Complex Ellipsoids, Complex Anal. Oper. Theory. DOI: 10.1007/s11785-015-0505-4 (2015).

[2] S. Bergman, Zur Theorie von pseudokonformen Abbildungen, Mat. Sb. (N.S.) 1(43)(1) (1936) 79-96.

[3] H.P. Boas, S. Fu and E.J. Straube, The Bergman kernel function: explicit formulas and zeroes, Proc. Amer. Math. Soc. 127(3) (1999) 805-811.

[4] J.P. D'Angelo, An explicit computation of the Bergman kernel function, $J$. Geom. Anal. 4 (1994) 23-34.

[5] A. Erdélyi, W. Magnus, F. Oberhettinger and F.G. Tricomi, Higher Transcendental Functions, (vol. 1, McGraw-Hill, New York, 1953).

[6] S. G. Krantz, Geometric analysis of the Bergman Kernel and Metric, (Springer, New York, 2013).

[7] D. D. Šiljak and D. M. Stipanović, Stability of Interval Two-Variable Polynomials and Quasipolynomials via Positivity, Lecture Notes in Control and Information Sciences. 312 (2005) 165-177.

[8] X. Wang, Recursion formulas for Appell functions, Integral Transforms Spec. Funct. 23(6) (2012) 421-433.

Tomasz Beberok

Department of Applied Mathematics

University of Agriculture in Krakow

ul. Balicka 253c, 30-198 Krakow, Poland

email: tbeberok@ar.krakow.pl 